PROCEEDINGS OF THE

AMERICAN MATHEMATICAL SOCIETY

Volume 135, Number 12, December 2007, Pages 3955-3965

S 0002-9939(07)09003-X

Article electronically published on June 19, 2007

\title{
SEPARATING CLASSES OF COMPOSITION OPERATORS VIA SUBNORMAL CONDITION
}

\author{
IL BONG JUNG, MI RYEONG LEE, AND SANG SOO PARK
}

(Communicated by Joseph A. Ball)

\begin{abstract}
Several classes have been considered to study the weak subnormalities of Hilbert space operators. One of them is $n$-hypnormality, which comes from the Bram-Halmos criterion for subnormal operators. In this note we consider $E(n)$-hyponormality, which is the parallel version corresponding to the Embry characterization for subnormal operators. We characterize $E(n)$ hyponormality of composition operators via $k$-th Radon-Nikodym derivatives and present some examples to distinguish the classes.
\end{abstract}

\section{INTRODUCTION}

Let $\mathcal{H}$ be an infinite-dimensional complex Hilbert space and let $\mathcal{L}(\mathcal{H})$ be the algebra of all bounded linear operators on $\mathcal{H}$. Several bridges of operators have been developed to detect how far from being subnormal a given hyponormal operator is. In 4, R. Curto defined strongly $n$-hyponormal operators by using the BramHalmos criterion, which states that an operator $T \in \mathcal{L}(\mathcal{H})$ is subnormal if and only if $\sum_{i, j}\left\langle T^{i} f_{j}, T^{j} f_{i}\right\rangle \geq 0$ for all finite collections $\left\{f_{i}\right\}$ in $\mathcal{H}$. Recall that $T$ is strongly $n$-hyponormal if $\sum_{i, j=0}^{n}\left\langle T^{i} f_{j}, T^{j} f_{i}\right\rangle \geq 0$ for any elements $f_{0}, f_{1}, \ldots, f_{n}$ in $\mathcal{H}(4)$. This notion has been studied to find gaps between subnormality and hyponormality of operators when their main models are weighted shifts (cf. [5, 6], [7, 19, 21, 22, 24]). Also those techniques in weighted shifts were useful to study $k$-contractive and $k$-hypercontractive operators (cf. [1, [13, [14]). In [10, they proved that every 2-hyponormal trigonometric Toeplitz operator is necessarily subnormal. An abstract solution to the lifting problem for commuting subnormal operators is obtained in [9] via $k$-hyponormality of multivariable weighted shifts. In [17] and [18] they show that strong $k$-hyponormality of a cyclic subnormal operator can be applied to a truncated complex moment problem by studying the flatness of moment matrices that was introduced in 8 .

An alternative characterization of subnormality is due to Embry ([1]), which is that an operator $T$ is subnormal if and only if $\sum_{i, j}\left\langle T^{i+j} f_{i}, T^{i+j} f_{j}\right\rangle \geq 0$ for all finite collections $\left\{f_{i}\right\}$ in $\mathcal{H}$. The following is defined as parallel with strong $n$-hyponormality.

Definition 1.1. For a positive integer $n$, an operator $T \in \mathcal{L}(\mathcal{H})$ is $E(n)$-hyponormal if $\sum_{i, j=0}^{n}\left\langle T^{i+j} f_{i}, T^{i+j} f_{j}\right\rangle \geq 0$ for any $f_{0}, f_{1}, \ldots, f_{n}$ in $\mathcal{H}$.

Received by the editors June 14, 2006 and, in revised form, November 7, 2006.

2000 Mathematics Subject Classification. Primary 47B20, 47B33; Secondary 47A63.

Key words and phrases. Composition operator, subnormal operator. 
It follows from [22] that for the case of weighted shifts, $n$-hyponormality and $E(n)$-hyponormality are equivalent, but the two notions are different in general. However, some computations show that if $T$ has dense range, then $T$ is $E(n)$ hyponormal if and only if $T$ is strongly $n$-hyponormal. Since a $(2 \times 2)$-operator matrix $\left(\begin{array}{cc}A & B \\ B^{*} & C\end{array}\right)$ (with $A$ invertible) is positive if and only if $A \geq 0, C \geq 0$, and $B^{*} A^{-1} B \leq C$, it is easy to see that an operator $T \in \mathcal{L}(\mathcal{H})$ is $E(1)$-hyponormal if and only if $\left|T^{2}\right|^{2} \geq|T|^{4}$. By the Löwner-Heinz inequality, every $E(1)$-hyponormal operator is always in the class $A$ (i.e., $\left|T^{2}\right| \geq|T|^{2}$ ) (cf. [15]). Note that every operator in the class $A$ is paranormal (cf. [16]). So $E(n)$-hyponormality provides a bridge between subnormal and paranormal operators in $\mathcal{L}(\mathcal{H})$.

Let $X:=(X, \mathcal{F}, \mu)$ be a $\sigma$-finite measure space and $T$ be a measurable transformation from $X$ into $X$, i.e., $T^{-1} \mathcal{F} \subset \mathcal{F}$. Let $C_{T}$ be a composition operator on $L^{2}:=L^{2}(X)$ defined by $C_{T} f=f \circ T$. In [3], they studied gaps between quasinormality and $p$-hyponormality via composition operators on $L^{2}$ for $p>0$. (Recall that $T$ is $p$-hyponormal if $\left(T^{*} T\right)^{p} \geq\left(T T^{*}\right)^{p}(p>0)$ and see the literature in [15] for further study). However, the information about the bridge of $E(n)$-hyponormal operators is not so well known, and so it is worthwhile to study $E(n)$-hyponormal composition operators on $L^{2}$. We will see that those two notions are different in Section 4.2.

This note consists of the following. In Section 2 we characterize the $E(n)$ hyponormal composition operators on $L^{2}$ as a positive matrix consisting of the $k$-th Radon-Nikodym derivatives generated by the absolutely continuous property $\mu \circ T^{-k} \ll \mu(k \leq 2 n)$. In Section 3 we obtain a formula separating the classes of $E(n)$-hyponormal composition operators on $L^{2}$. In Section 4 we provide a useful related example.

\section{2. $E(n)$-HYPONORMAL COMPOSITION OPERATORS}

Let $X:=(X, \mathcal{F}, \mu)$ be a $\sigma$-finite measure space and let $T$ be a non-singular transformation on $X$ (i.e., $\mu \circ T^{-1} \ll \mu$ ) throughout this paper. Since $\mu$ is $\sigma$ finite, there exists a measurable non-negative extended real-valued function (RadonNikodym derivative) $h_{1}$ such that $\mu \circ T^{-1}(A)=\int_{A} h_{1} d \mu$, for all $A$ in $\mathcal{F}$. More generally, since $\mu \circ T^{-k} \ll \mu(k \geq 2)$, we have the $k$-th Radon-Nikodym derivative

$h_{k}$ defined by $\mu \circ T^{-k}(A)=\int_{A} h_{k} d \mu$, for all $A$ in $\mathcal{F}$, i.e., $h_{k}:=\frac{d \mu \circ T^{-k}}{d \mu}$ (cf. [3]). For a convention we write $h_{0}(x)=1$. We now begin our work with the following lemma.

Lemma 2.1. Let $\left\{\phi_{i j}\right\}_{i, j=0}^{n}$ be a subset of functions in $L^{\infty}$ such that $\phi_{i j}(x)=$ $\overline{\phi_{j i}(x)}$. Assume that, for any $f_{i} \in L^{2}, 0 \leq i \leq n$,

$$
\sum_{i, j=0}^{n} \int_{X} \phi_{j i}(x) \cdot f_{i}(x) \cdot \overline{f_{j}(x)} d \mu \geq 0
$$

Then, for any $f_{i} \in L^{2}, 1 \leq i \leq n$,

$$
\sum_{i, j=1}^{n} \int_{X}\left(\phi_{00}(x) \phi_{j i}(x)-\phi_{i 0}(x) \phi_{0 j}(x)\right) \cdot f_{i}(x) \cdot \overline{f_{j}(x)} d \mu \geq 0 .
$$


Proof. Assuming the contrary, we suppose there exist $f_{j} \in L^{2}, 1 \leq j \leq n$, such that

$$
\sum_{i, j=1}^{n} \int_{X}\left(\phi_{00}(x) \phi_{j i}(x)-\phi_{i 0}(x) \phi_{0 j}(x)\right) \cdot f_{i}(x) \cdot \overline{f_{j}(x)} d \mu<0 .
$$

Let $A_{n}:=\left\{x \in X \mid \phi_{00}(x) \geq 1 / n\right\}$ and let $\chi_{A_{n}}$ be the characteristic function on $A_{n}$. Since $\phi_{00}(x) \geq 0$ a.e. $x$ in $X$, by the Lebesgue dominated convergence theorem, there exists a number $n_{0} \in \mathbb{N}$ satisfying

$$
\sum_{i, j=0}^{n} \int_{X}\left(\phi_{00}(x) \phi_{j i}(x)-\phi_{i 0}(x) \phi_{0 j}(x)\right) \cdot f_{i}(x) \cdot \overline{f_{j}(x)} \chi_{A_{n_{0}}} d \mu<0 .
$$

Now let $g_{j}=\chi_{A_{n_{0}}} f_{j}, 1 \leq j \leq n$, and define $g_{0}$ by

$$
g_{0}(x)=\left\{\begin{array}{cl}
-\frac{1}{\phi_{00}(x)}\left(\phi_{01}(x) g_{1}(x)+\cdots+\phi_{0 n}(x) g_{n}(x)\right), & \text { if } x \in A_{n_{0}}, \\
0, & \text { if } x \notin A_{n_{0}} .
\end{array}\right.
$$

Then, $g_{0} \in L^{2}$ and $\phi_{00}(x) g_{0}(x)+\phi_{01}(x) g_{1}(x)+\cdots+\phi_{0 n}(x) g_{n}(x)=0$, for all $x \in X$. Hence we have $\sqrt{\phi_{00}} g_{j} \in L^{2}, 0 \leq j \leq n$, but

$$
\begin{aligned}
\sum_{i, j=0}^{n} \int_{X} \phi_{j i}(x)\left(\sqrt{\phi_{00}} g_{i}\right)(x) \cdot\left(\overline{\sqrt{\phi_{00}} g_{j}}\right)(x) d \mu \\
=\int_{X}\left|\phi_{00}(x) g_{0}(x)+\phi_{01}(x) g_{1}(x)+\cdots+\phi_{0 n}(x) g_{n}(x)\right|^{2} d \mu \\
\quad+\sum_{i, j=1}^{n} \int_{X}\left(\phi_{00}(x) \phi_{j i}(x)-\phi_{i 0}(x) \phi_{0 j}(x)\right) \cdot g_{i}(x) \cdot \overline{g_{j}(x)} d \mu \\
=\sum_{i, j=1}^{n} \int_{X}\left(\phi_{00}(x) \phi_{j i}(x)-\phi_{i 0}(x) \phi_{0 j}(x)\right) \cdot f_{i}(x) \overline{f_{j}(x)} \chi_{A_{n_{0}}}(x) d \mu<0,
\end{aligned}
$$

which leads to a contradiction.

Lemma 2.2. Let $\phi_{i j} \in L^{\infty}$ such that $\phi_{i j}(x)=\overline{\phi_{j i}(x)}$ for $0 \leq i, j \leq n$. Then the following assertions are equivalent:

(i) for any $f_{i} \in L^{2}, 0 \leq i \leq n$,

$$
\sum_{i, j=0}^{n} \int_{X} \phi_{j i}(x) \cdot f_{i}(x) \cdot \overline{f_{j}(x)} d \mu \geq 0
$$

(ii) the $(n+1) \times(n+1)$ complex matrix $\left(\phi_{i j}(x)\right)_{i, j=0}^{n}$ is (pointwise) positive for a.e. $x$ in $X$ w.r.t. $\mu$.

Proof. Since (ii) $\Rightarrow$ (i) is obvious, it is sufficient to show that (i) $\Rightarrow$ (ii). First we claim that $\phi_{00}(x) \geq 0$ a.e. To the contrary, we suppose that there exists $\epsilon>0$ such that $\phi_{00}(x)<-\epsilon$ on a positive measurable set $E$. Let $f_{0}(x)=\chi_{E}(x)$. Then we have

$$
\int_{X} \phi_{00}(x)\left|f_{0}(x)\right|^{2} d \mu<-\epsilon \cdot \mu(E)<0,
$$

which is a contradiction of (2.1). Note that this is the case $n=0$ in the mathematical induction. We assume that the implication holds for the case $n=k-1$. If (i) 
holds in the case of $n=k$, i.e.,

$$
\sum_{i, j=0}^{k} \int_{X} \phi_{j i}(x) \cdot f_{i}(x) \cdot \overline{f_{j}(x)} d \mu \geq 0
$$

then it follows from Lemma 2.1 that for any $f_{i} \in L^{2}, 1 \leq i \leq k$,

$$
0 \leq \sum_{i, j=1}^{k} \int_{X}\left(\phi_{00}(x) \phi_{j i}(x)-\phi_{i 0}(x) \phi_{0 j}(x)\right) \cdot f_{i}(x) \cdot \overline{f_{j}(x)} d \mu .
$$

Then the hypothesis of mathematical induction shows that

$$
\left(\phi_{00}(x) \phi_{j i}(x)-\phi_{j 0}(x) \phi_{0 i}(x)\right)_{i, j=1}^{k} \geq 0 .
$$

If $x$ satisfies $\phi_{00}(x) \neq 0$, then obviously we have

$$
\left(\phi_{j i}(x)-\frac{\phi_{j 0}(x) \phi_{0 i}(x)}{\phi_{00}(x)}\right)_{i, j=1}^{k} \geq 0,
$$

which is equivalent to $\left(\phi_{j i}(x)\right)_{i, j=0}^{k} \geq 0$.

In the case that $N:=\left\{x: \phi_{00}(x)=0\right\}$ is a non-zero measurable set, it follows immediately from (2.3) that $\phi_{j 0}(x)=0$ for $x \in N$ a.e., $1 \leq j \leq k$. Moreover, applying $f_{0}(x) \equiv 0$ to $(2.2)$, we have that for any $f_{j} \in L^{2}, 1 \leq j \leq k$,

$$
\sum_{i, j=1}^{k} \int_{X} \phi_{j i}(x) f_{i}(x) \overline{f_{j}(x)} d \mu \geq 0 .
$$

By the hypothesis of mathematical induction, it follows that $\left(\phi_{i j}(x)\right)_{i, j=1}^{k} \geq 0$ a.e. Hence the condition $\phi_{i 0}(x)=0(0 \leq i \leq k)$ yields that $\left(\phi_{i j}(x)\right)_{i, j=0}^{k} \geq 0$, which completes the proof.

Theorem 2.3. Suppose $n \in \mathbb{N}$. Let $C_{T}$ be a composition operator on $L^{2}$. Then $C_{T}$ is $E(n)$-hyponormal if and only if the $(n+1) \times(n+1)$ complex matrix $\Delta_{n}(x) \geq 0$ for a.e. $x$ w.r.t. $\mu$, where

$$
\Delta_{n}(x):=\left(\begin{array}{cccc}
h_{0}(x) & h_{1}(x) & \cdots & h_{n}(x) \\
h_{1}(x) & h_{2}(x) & \cdots & h_{n+1}(x) \\
\vdots & \vdots & \ddots & \vdots \\
h_{n}(x) & h_{n+1}(x) & \cdots & h_{2 n}(x)
\end{array}\right) .
$$

Proof. Since $\left(C_{T}^{n *} C_{T}^{n}\right) f=h_{n} f$ for all $n \in \mathbb{N}$ and any $f$ in $L^{2}$, we have that

$$
\left\langle C_{T}^{* i+j} C_{T}^{i+j} f_{i}, f_{j}\right\rangle=\int_{X} h_{i+j}(x) f_{i}(x) \overline{f_{j}(x)} d \mu \quad\left(f_{i} \in L^{2}, 0 \leq i \leq n\right) .
$$

Hence by Lemma 2.2, we have this theorem.

According to Theorem 2.3, we have that $C_{T}$ is subnormal if and only if $\Delta_{n}(x) \geq 0$ a.e. $x$ and for all $n \in \mathbb{N}_{0}:=\mathbb{N} \cup\{0\}$ if and only if $\left\{h_{n}(x)\right\}_{n=0}^{\infty}$ is a moment sequence a.e. $x$. The following is an immediate corollary of Theorem 2.3 .

Corollary 2.4. Let $C_{T}$ be a composition operator on $L^{2}$. Then $C_{T}$ is $E(1)$ hyponormal if and only if $h_{2}(x) \geq h_{1}(x)^{2}$ for a.e. $x$ in $X$ if and only if $C_{T}$ is paranormal. 
Proof. Let $E(f):=E\left(f \mid T^{-1} \mathcal{F}\right)$ be the conditional expectation of $f$ in $L^{2}$ with respect to $T^{-1} \mathcal{F}$ (cf. [3], 23]). Recall that $E\left(h_{1}\right) \geq h_{1} \circ T$ if and only if $C_{T}$ is paranormal (2]). Since $h_{2}=h_{1} E\left(h_{1}\right) \circ T^{-1}$ (cf. [20]), a simple computation proves that if $C_{T}$ is paranormal, then $h_{2}(x) \geq h_{1}(x)^{2}$.

The following is a generalization of recursively generated weighted shifts (whose definition will appear in the next section) corresponding to composition operators.

Corollary 2.5. Let $C_{T}$ be a composition operator on $L^{2}$ and let $k \in \mathbb{N}$. Assume that there exist $\psi_{j} \in L^{\infty}$ for $j=0, \ldots, k-1$, such that

$$
h_{k+i}(x)=\psi_{k-1}(x) h_{k-1+i}(x)+\psi_{k-2+i}(x) h_{k-2+i}(x)+\cdots+\psi_{0}(x) h_{i}(x)
$$

for a.e. $x$ in $X$ and $i=0,1,2, \ldots$ Let $r$ be the the smallest positive integer satisfying $(2.6 r)$. Then $C_{T}$ is E(r)-hyponormal if and only if $C_{T}$ is subnormal.

Proof. Suppose $C_{T}$ is $E(r)$-hyponormal. Then, by the recursive relation $(2.6 k)$, rank $\Delta_{m}(x)=\operatorname{rank} \Delta_{m+1}(x)$ a.e. $x$ in $X$, for all $m \geq r$, and hence $\Delta_{m}(x) \geq 0$ a.e. $x$ in $X$ for all $m \geq r$ (cf. [6. Prop. 2.3]). So this corollary follows from Theorem 2.3 .

The operator appearing in Corollary 2.5 is referred to as "a recursively generated composition operator with rank $r$ " (cf. [6]). An example satisfying the hypothesis of Corollary 2.5 will be introduced in Section 4.

Proposition 2.6. Suppose that $C_{T}$ has dense range and $h_{1}>0$. Let $n \in \mathbb{N}$. Then $C_{T}^{*}$ is $E(n)$-hyponormal if and only if the $(n+1) \times(n+1)$ complex matrix

$$
\Gamma_{n}(x):=\left(\begin{array}{cccc}
h_{0}(x) & h_{1} \circ T(x) & \cdots & h_{n} \circ T^{n}(x) \\
h_{1} \circ T(x) & h_{2} \circ T^{2}(x) & \cdots & h_{n+1} \circ T^{n+1}(x) \\
\vdots & \vdots & \ddots & \vdots \\
h_{n} \circ T^{n}(x) & h_{n+1} \circ T^{n+1}(x) & \cdots & h_{2 n} \circ T^{2 n}(x)
\end{array}\right)
$$

is positive a.e. $x$ in $X$ w.r.t. $\mu$.

Proof. Let $E_{n} f=E\left(f \mid T^{-n} \mathcal{F}\right)$ be the conditional expectation of $f$ in $L^{2}$ with respect to $T^{-n} \mathcal{F}$ for $n \geq 1$. Then it follows from [12] that $C^{n} C^{* n} f=h_{n} \circ T^{n} E_{n} f$ $\left(f \in L^{2}, n \in \mathbb{N}\right)$. Hence, we have that

$$
\sum_{i, j=0}^{n}\left\langle C^{i+j} C^{* i+j} f_{i}, f_{j}\right\rangle \geq 0 \Longleftrightarrow \sum_{i, j=0}^{n}\left\langle h_{i+j} \circ T^{i+j} E_{i+j} f_{i}, f_{j}\right\rangle \geq 0,
$$

for any $f_{i} \in L^{2}, 0 \leq i \leq n$. Since $T^{-1} \mathcal{F}=\mathcal{F}$, each of the above inequalities is equivalent to

$$
\sum_{i, j=0}^{n}\left\langle h_{i+j} \circ T^{i+j} f_{i}, f_{j}\right\rangle \geq 0
$$

that is,

$$
\sum_{i, j=0}^{n} \int_{X} h_{i+j} \circ T^{i+j}(x) f_{i}(x) \cdot \overline{f_{j}(x)} d \mu \geq 0 .
$$

By Lemma 2.2, (2.7) is equivalent to $\Gamma_{n}(x) \geq 0$ a.e. $x$ in $X$.

We close this section with the following remark. 
Remark 2.7. It is well known that $T^{-1} \mathcal{F}=\mathcal{F}$ if and only if $C_{T}$ has dense range (cf. [26]). Thus, if $T^{-1} \mathcal{F}=\mathcal{F}$, then $C_{T}$ is strongly $n$-hyponormal if and only if $C_{T}$ is $E(n)$-hyponormal. It is worthwhile to find characterizations of strong $n$ hyponormality [or $E(n)$-hyponormality] of $C_{T}$ [or $C_{T}^{*}$, resp.] without the hypothesis in Proposition 2.6. However we do not yet obtain a useful result about them.

\section{Separating Classes of $E(n)$-hyponormal operators}

Let $\gamma:=\left\{\gamma_{n}\right\}_{n=0}^{\infty}$ be a set of positive real numbers. For brevity we consider $\gamma_{0}=1$ in this section. A sequence $\gamma$ is said to be recursively generated if there exist a natural number $r$ and a vector $\psi=\left(\psi_{0}, \ldots, \psi_{r-1}\right) \in \mathbb{C}^{r}$ such that $\gamma_{n}=$ $\psi_{r-1} \gamma_{n-1}+\cdots+\psi_{0} \gamma_{n-r}(n \geq r)$. A sequence $\gamma$ is non-recursively generated if it is not recursively generated. (Note that the weighted shift $W_{\alpha}$ with weight sequence $\alpha_{n}:=\sqrt{\gamma_{n+1} / \gamma_{n}}$ is called recursively generated. The numbers $\gamma_{0}:=1$ and $\gamma_{n}:=\alpha_{0}^{2} \cdots \alpha_{n-1}^{2}(n \geq 1)$ are called moments of $W_{\alpha}$ in [6, Prop. 2.6]).

Suppose $\left\{\gamma_{k}\right\}_{k=0}^{\infty}$ is a non-recursively generated sequence. Let

$$
A(i, j)=\left(\begin{array}{ccccc}
\gamma_{i} & \gamma_{i+1} & \gamma_{i+2} & \cdots & \gamma_{i+j} \\
\gamma_{i+1} & \gamma_{i+2} & \gamma_{i+3} & \cdots & \gamma_{i+j+1} \\
\vdots & \vdots & \vdots & \ddots & \vdots \\
\gamma_{i+j} & \gamma_{i+j+1} & \gamma_{i+j+2} & \cdots & \gamma_{i+2 j}
\end{array}\right)
$$

for $i, j \in \mathbb{N}_{0}$. If $A(i, j) \geq 0$, the non-recursivity of $\left\{\gamma_{k}\right\}_{k=0}^{\infty}$ implies that $\operatorname{det} A(i, j)>$ $0\left(i, j \in \mathbb{N}_{0}\right)$ (cf. [6, Prop. 2.6], [19]). Let

$$
\Psi_{k}(t)=\left(\begin{array}{ccccc}
t & \gamma_{0} & \gamma_{1} & \cdots & \gamma_{k-1} \\
\gamma_{0} & \gamma_{1} & \gamma_{2} & \cdots & \gamma_{k} \\
\vdots & \vdots & \vdots & \ddots & \vdots \\
\gamma_{k-1} & \gamma_{k} & \gamma_{k+1} & \cdots & \gamma_{2 k-1}
\end{array}\right)
$$

where $t$ is a positive real variable. Then $\operatorname{det} \Psi_{k}(t)$ is a polynomial in $t$ of degree 1 and has the unique zero $\xi_{k}>0$.

Lemma 3.1 ([19, Th. 2.1]). Under the same notation and hypothesis as above, it follows that

$$
\xi_{k+1}-\xi_{k}=\frac{(\operatorname{det} A(0, k))^{2}}{\operatorname{det} A(1, k-1) \cdot \operatorname{det} A(1, k)}>0 \quad(k \geq 1) .
$$

Let $X:=\mathbb{N}_{0}$ and let $\mathcal{F}:=\mathcal{P}(X)$ be the power set of $X$. We define a measurable transformation $T$ on $X$ by $T(k)=k-1(k \geq 1)$ and $T(0)=0$. Let $m(k)=$ $m_{k} \in \mathbb{R}^{+}$be an arbitrary point mass measure on $X$ with $m_{0}=t$ as a positive real variable. Then the $\sigma$-algebra $T^{-1} \mathcal{F}$ is generated by atoms $\{0,1\},\{2\},\{3\}, \cdots$. By a computation, we obtain the Radon-Nikodym derivative $h_{1}=\frac{d \mu \circ T^{-1}}{d \mu}$ :

$$
h_{1}(0)=\frac{\mu \circ T^{-1}(0)}{\mu(0)}=\frac{t+m_{1}}{t} \text { and } h_{1}(k)=\frac{m_{k+1}}{m_{k}} \quad(k \geq 1),
$$

i.e., $h_{1}$ is a sequence of the form: $\frac{t+m_{1}}{t}, \frac{m_{2}}{m_{1}}, \frac{m_{3}}{m_{2}}, \frac{m_{4}}{m_{3}}, \cdots$. By simple computations, we also have that for each $n \geq 1$,

$$
h_{n}(0)=\frac{t+m_{1}+\cdots+m_{n}}{t} \text { and } h_{n}(k)=\frac{\mu \circ T^{-n}(\{k\})}{\mu(\{k\})}=\frac{m_{n+k}}{m_{k}} \quad(k \geq 1) .
$$

Then we have the following lemma. 
Lemma 3.2. Let $\left\{m_{k}\right\}_{k=0}^{\infty}$ be the sequence as above. Then we have $\Delta_{n}(0)=$ $\left(h_{i+j}(0)\right)_{i, j=0}^{n}=\frac{1}{t}\left(d_{i j}\right)_{i, j=0}^{n}$, where $d_{i j}=\sum_{p=0}^{i+j} m_{p}$.

Lemma 3.3. Let $\left\{m_{k}\right\}_{k=0}^{\infty}$ be the sequence as above. For $n \in \mathbb{N}$, we let

$$
\Theta_{n}(t)=\left(\begin{array}{ccccc}
t & \beta_{0} & \beta_{1} & \cdots & \beta_{n-1} \\
\beta_{0} & \beta_{1} & \beta_{2} & \cdots & \beta_{n} \\
\vdots & \vdots & \vdots & \ddots & \vdots \\
\beta_{n-1} & \beta_{n} & \beta_{n+1} & \cdots & \beta_{2 n-1}
\end{array}\right)
$$

with

$$
\beta_{k}=\sum_{i=0}^{k}(-1)^{i}\left(\begin{array}{c}
k \\
i
\end{array}\right) m_{k+1-i} .
$$

Then $\operatorname{det}\left(d_{i j}\right)_{i, j=0}^{n}=\operatorname{det} \Theta_{n}(t)$.

Proof. According to the elementary row operations and column operations, without difficulty we may obtain the required equality.

With the same notation as in (2.5) and (3.3), we denote

$$
R E(n, k)=\left\{t>0: \Delta_{n}(k, t) \geq 0\right\},
$$

where $\Delta_{n}(k, t) \equiv \Delta_{n}(k)$ is as in $(2.5)$, and

$$
R D(n, k)=\left\{t>0: \operatorname{det} \Delta_{i}(k, t)>0,1 \leq i \leq n-1 \text { and } \operatorname{det} \Delta_{n}(k, t) \geq 0\right\}
$$

for $n=1,2, \cdots$. Then we have the following lemma.

Lemma 3.4. Let $\left\{m_{k}\right\}_{k=0}^{\infty}$ be a sequence of positive real numbers with $m_{0}=t$ as a positive real variable such that $\left\{\frac{m_{n+k}}{m_{k}}\right\}_{n=0}^{\infty}$ is a non-recursively generated sequence for any $k \in \mathbb{N}$. Let $\beta_{k}$ be as in (3.4) and let $\xi_{n}$ be the zero of $\operatorname{det} \Theta_{n}(t)$. Then we have the following assertions:

(i) $R E(n, k)=R D(n, k)$ for all $n=1,2, \cdots$ and $k \in \mathbb{N}_{0}$;

(ii) $R E(n, k) \backslash R E(n+1, k)=\left[\xi_{n}, \xi_{n+1}\right)\left(n \geq 1, k \in \mathbb{N}_{0}\right)$;

(iii) $\bigcap_{n=1}^{\infty} R E(n, k)=\left[\xi_{\infty}, \infty\right)$ for any $k \in \mathbb{N}_{0}$, where $\xi_{\infty}=\lim \xi_{n}$.

Proof. By the Nested Determinants Test $([\underline{6})$, obviously we have that $R E(n, k) \supset$ $R D(n, k)$. To prove the reverse containment, let $t \in R E(n, k)$, i.e., $\Delta_{n}(k, t) \geq 0$. Since $\left\{\frac{m_{n+k}}{m_{k}}\right\}_{n=0}^{\infty}$ is non-recursively generated, $\operatorname{det}\left(\frac{m_{i+j+k}}{m_{k}}\right)_{i, j=0}^{n}>0$ for any $k \in \mathbb{N}$. Hence $R E(n, k) \subset R D(n, k)=(0, \infty), k \in \mathbb{N}$. So we only consider $k=0$. For a contradiction, we suppose that there exists $n_{0} \in \mathbb{N}$ with $1 \leq n_{0} \leq n-1$ such that $\Delta_{n_{0}}(0, t) \geq 0$ and $\operatorname{det} \Psi_{n_{0}}(t)=0$ for all $t>0$. (Note that $\operatorname{det} \Theta_{n_{0}}(t)=$ $\operatorname{det} \Delta_{n_{0}}(0, t)$ by Lemma 3.3.) Then it follows from [6] that

$$
\operatorname{det} \Theta_{n_{0}}(t)=0=\operatorname{det} \Theta_{n_{0}+1}(t)=\cdots=\operatorname{det} \Theta_{n}(t) \quad(t>0) .
$$

So we can find a root $\xi_{n_{0}}$ satisfying $\operatorname{det} \Theta_{n_{0}}\left(\xi_{n_{0}}\right)=0$. Since the slope of $\operatorname{det} \Theta_{n_{0}}(x)$ is positive and $\xi_{n_{0}}<\xi_{n_{0}+1}$, we have $\operatorname{det} \Theta_{n_{0}+1}\left(\xi_{n_{0}}\right)<0$, which contradicts $\Delta_{n}(0, k) \geq$ 0 . Hence $R E(n, 0) \subset R D(n, 0)$. The remaining parts are routine.

By the above lemmas and Theorem 2.3, we have the following theorem. 
Theorem 3.5. Consider a measure space $X:=\mathbb{N}_{0}$ and the power set of $\mathbb{N}_{0}$ as a $\sigma$-algebra $\mathcal{F}:=\mathcal{P}(X)$. Let $m(k)=m_{k}$ be an arbitrary point mass measure on $(X, \mathcal{F})$ with positive real variable $m_{0}=t$ and let $T$ be a measurable transformation on $X$ defined by $T(k)=k-1(k \geq 1)$ and $T(0)=0$. Assume that $\left\{\frac{m_{n+k}}{m_{k}}\right\}_{n=0}^{\infty}$ is a non-recursive sequence for any $k \in \mathbb{N}$. Let $\beta_{k}$ be as in (3.4) and let $\xi_{k}$ be the zero of $\operatorname{det} \Theta_{k}(t)$ with $\beta_{k}=\sum_{i=0}^{k}(-1)^{i}\left(\begin{array}{c}k \\ i\end{array}\right) m_{k+1-i}$. Then

(i) $\left\{\xi_{k}\right\}_{k=1}^{\infty}$ is a strictly increasing sequence of positive real numbers;

(ii) $R E(n, 0)=\left\{t>0: C_{T}\right.$ is $E(n)$-hyponormal $\}=\left[\xi_{n}, \infty\right)$;

(iii) $R E(n, 0) \backslash R E(n+1,0)=\left[\xi_{n}, \xi_{n+1}\right) \quad(n \geq 1)$;

(iv) $\left\{t>0: C_{T}\right.$ is subnormal $\}=\left[\xi_{\infty}, \infty\right)$.

We close this section with an example for Theorem 3.5.

Example 3.6. Let $(X, \mathcal{F})$ be as in Theorem 3.5 and let a measure $m(k)$ on $X$ be defined by

$$
m(k) \equiv m_{k}=\int_{1}^{2}\left(\lambda^{k}-\lambda^{k-1}\right) d \lambda, \quad k \geq 1 .
$$

Let $C_{T}$ be a composition operator defined as in Theorem 3.5. Since the moment measure $d \lambda$ has infinite support, $\left\{\frac{m_{n+k}}{m_{k}}\right\}_{n=0}^{\infty}$ is non-recursive for any $k \in \mathbb{N}$ (cf. 25), p.6]). By simple computations, we obtain $\xi_{n}=\frac{n(n+2)}{(n+1)^{2}}$. Therefore

$$
C_{T} \text { is } E(n) \text {-hyponormal } \Longleftrightarrow \frac{n(n+2)}{(n+1)^{2}} \leq t<\infty .
$$

It follows from [3, Prop. 2.4] that $C_{T}$ is hyponormal if and only if $h_{1}>0$ and $E\left(1 / h_{1}\right) \leq 1 /\left(h_{1} \circ T\right)$, where $E$ is the usual conditional expectation, which is equivalent to $\frac{3}{4} \leq t<\infty$. Hence $C_{T}$ is hyponormal if and only if it is $E(1)$ hyponormal. Moreover, $C_{T}$ is subnormal if and only if $1 \leq t<\infty$.

\section{Further EXAmples}

In this section, we will discuss two examples.

4.1. Let $r$ be any fixed non-negative integer and let $\eta=\sum_{i=0}^{r} \rho_{i} \delta_{\left\{p_{i}\right\}}$ be a positive measure on $X:=\mathbb{N}_{0}$ for some densities $\rho_{i}>0$ and $p_{i} \in \mathbb{N}_{0}$. Define $m_{0}=x>0$ and $m_{k}=\int t^{k+1} d \eta(k \in \mathbb{N})$. Let $T$ be a measurable transformation on $(X, \mathcal{P}(X))$ defined by $T(k)=0$ if $k=0,1$, and $T(k)=k-1$ otherwise. Let $q_{i}(0 \leq i \leq r)$ be the coefficient of $t^{i}$ in the polynomial $\prod_{j=0}^{r}\left(t-p_{j}\right)$. Since

$$
\sum_{j=0}^{r} q_{j} m_{j+k}=\int \sum_{j=0}^{r} q_{j} t^{j+k+1} d \eta=\int t^{k+1} \prod_{j=0}^{r}\left(t-\rho_{j}\right) d \eta=0, \text { for } k \geq 1,
$$

we have that for $k \geq 1$,

$$
h_{r+n}(k)=\frac{m_{r+n+k}}{m_{k}}=-\frac{1}{m_{k}} \sum_{j=0}^{r-1} q_{j} m_{j+n+k}=-\sum_{j=0}^{r-1} q_{j} h_{j+n}(k)\left(n \in \mathbb{N}_{0}\right) .
$$

We want to show that

$$
\begin{aligned}
h_{r+n+1}(0)= & \left(1-q_{r-1}\right) h_{r+n}(0)+\left(q_{r-1}-q_{r-2}\right) h_{r+n-2}(0) \\
& +\cdots+\left(q_{1}-q_{0}\right) h_{n}(0)+q_{0} h_{n-1}(0) .
\end{aligned}
$$


If we set $s_{k}:=\sum_{j=0}^{k} m_{j}$, then we have, for $n \in \mathbb{N}_{0}$,

$$
\begin{aligned}
s_{r+n+1} & =s_{r+n}+m_{r+n+1}=s_{r+n}-\sum_{j=0}^{r-1} q_{j} m_{j+n+1}=s_{r+n}-\sum_{j=0}^{r-1} q_{j}\left(s_{j+n+1}-s_{j+n}\right) \\
& \left.=1-q_{r-1}\right) s_{r+n}+\left(q_{r-1}-q_{r-2}\right) s_{r+n-1}+\cdots+\left(q_{1}-q_{0}\right) s_{n+1}+q_{0} s_{n} .
\end{aligned}
$$

Using the fact that $h_{r+n+1}(0)=\frac{s_{r+n+1}}{m_{0}}$, it follows that

$$
\begin{aligned}
h_{r+n+1}(0) & =\left(1-q_{r-1}\right) h_{r+n}(0)+\left(q_{r-1}-q_{r-2}\right) h_{r+n-1}(0) \\
& +\cdots+\left(q_{1}-q_{0}\right) h_{n+1}(0)+q_{0} h_{n}(0) .
\end{aligned}
$$

Thus, by (4.1) and (4.2), $C_{T}$ is a recursively generated composition operator satisfying $(2.6 k)$ in Corollary 2.5 with rank less than or equal to $r+1$. Hence we obtain that $C_{T}$ is $E(r+1)$-hyponormal if and only if $C_{T}$ is subnormal.

In particular, let us consider $\eta:=\rho_{0} \delta_{\left\{p_{0}\right\}}+\rho_{1} \delta_{\left\{p_{1}\right\}}$ satisfying $\int t d \eta>\int d \eta$, $p_{0} \neq 1$, and $p_{1} \neq 1$. We denote $R E(n):=\left\{x>0: C_{T}\right.$ is $E(n)$-hyponormal $\}$. Then,

$$
R E(1)=\left\{x: x \geq\left(\int 1 d \eta\right)^{2}\left(\int(t-1) d \eta\right)^{-1}\right\} .
$$

It follows from the above fact that $R E(2)=R E(3)=\cdots=R E(\infty):=\bigcap_{n=0}^{\infty} R E(n)$. At first observe that

$$
x+\int\left(1+\cdots+t^{n}\right) d \eta=x-\int \frac{1}{t-1} d \eta+\int \frac{t^{n+1}}{t-1} d \eta .
$$

Therefore we can easily verify that for any $a, b, c \in \mathbb{C}$,

$$
\begin{array}{r}
\left\langle\Delta_{2}(0)\left(\begin{array}{l}
a \\
b \\
c
\end{array}\right),\left(\begin{array}{l}
a \\
b \\
c
\end{array}\right)\right\rangle=\left\langle\Omega\left(\begin{array}{l}
a \\
b \\
c
\end{array}\right),\left(\begin{array}{l}
a \\
b \\
c
\end{array}\right)\right\rangle \\
=\left(x-\int \frac{1}{t-1} d \eta\right)|a+b+c|^{2}+\int \frac{\left|a+b t+b t^{2}\right|^{2}}{t-1} d \eta,
\end{array}
$$

where

$$
\Omega:=\left(\begin{array}{ccc}
x & x+\int d \eta & x+\int(1+t) d \eta \\
x+\int d \eta & x+\int(1+t) d \eta & x+\int\left(1+t+t^{2}\right) d \eta \\
x+\int(1+t) d \eta & x+\int\left(1+t+t^{2}\right) d \eta & x+\int\left(1+t+t^{2}+t^{3}\right) d \eta
\end{array}\right) .
$$

Hence $C_{T}$ is $E(2)$-hyponormal if and only if $x \geq \int \frac{1}{t-1} d \eta$, which also is equivalent to $C_{T}$ being subnormal.

4.2. In this subsection we give examples showing that the notions of $E(n)$-hyponormality and $p$-hyponormality are different as we promised in the introduction. Let $\alpha: 1,1,2,2, \ldots$ be a weight sequence and let $W_{\alpha}$ be its corresponding weighted shift. Then obviously $W_{\alpha}$ is $p$-hyponormal for $p>0$. However $W_{\alpha}$ is $E(1)$-hyponormal but not $E(k)$-hyponormal for any $k \geq 2$. Now we consider $T=I_{\mathcal{H}}+S$, where $S$ is the unilateral unweighted shift of multiplicity one. Then obviously $T$ is subnormal, and thus $T$ is $E(n)$-hyponormal for all $n \in \mathbb{N}$. By some computations, we have that

$$
|T|^{2(1+\varepsilon)}-\left|T^{*}\right|^{2(1+\varepsilon)}=\left(\begin{array}{cc}
t_{1}(\varepsilon) & t_{2}(\varepsilon) \\
t_{2}(\varepsilon) & t_{3}(\varepsilon)
\end{array}\right) \oplus 0_{\mathcal{H} \ominus \mathbb{C}^{2}} \quad(\varepsilon>0),
$$


where

$$
\begin{aligned}
& t_{1}(\varepsilon)=\frac{1+3^{1+\varepsilon}}{2}+\frac{-5+\sqrt{5}}{10}\left(\frac{3-\sqrt{5}}{2}\right)^{\varepsilon}-\frac{5+\sqrt{5}}{10}\left(\frac{3+\sqrt{5}}{2}\right)^{\varepsilon}, \\
& t_{2}(\varepsilon)=\frac{-1+3^{1+\varepsilon}}{2}+\frac{-5+3 \sqrt{5}}{10}\left(\frac{3-\sqrt{5}}{2}\right)^{\varepsilon}-\frac{5+3 \sqrt{5}}{10}\left(\frac{3+\sqrt{5}}{2}\right)^{\varepsilon}, \\
& t_{3}(\varepsilon)=\frac{1+3^{1+\varepsilon}}{2}-\frac{5-2 \sqrt{5}}{5}\left(\frac{3-\sqrt{5}}{2}\right)^{\varepsilon}-\frac{5+2 \sqrt{5}}{5}\left(\frac{3+\sqrt{5}}{2}\right)^{\varepsilon} .
\end{aligned}
$$

It can be easily verified that $\operatorname{det}\left(\begin{array}{cc}t_{1}(\varepsilon) & t_{2}(\varepsilon) \\ t_{2}(\varepsilon) & t_{3}(\varepsilon)\end{array}\right)<0$ for all $\varepsilon>0$, and hence $T$ is not $(1+\varepsilon)$-hyponormal for any $\varepsilon>0$. Therefore " $E(n)$-hyponormality is independent from $p$-hyponormality for $p>0$ ".

\section{ACKNOWLEDGMENTS}

The first author was supported by the Korea Research Foundation Grant funded by the Korean Government (MOEHRD) (KRF-2006-312-C00027). The third author was supported by a grant, KRF-2004-050-C00003. We express our thanks to the referee for several excellent remarks that allowed us to correct some minor errors and to improve the exposition herein.

\section{REFERENCES}

[1] J. Agler, Hypercontractions and subnormality, J. Operator Theory, 13(1985), 203-217. MR775993 (86i:47028)

[2] C. Burnap and I. Jung, Composition operators with weak hyponormality, J. Math. Anal. Appl., to appear.

[3] C. Burnap, I. Jung and A. Lambert, Separating partial normality classes with composition operators, J. Operator Theory, 53(2005), 381-397. MR.2153155

[4] R. Curto, Quadratically hyponormal weighted shifts, Integral Equation Operator Theory 13(1990), 49-66. MR1025673 (90k:47061)

[5] Joint hyponormality: A bridge between hyponormality and subnormality, Proc. Sympos. Math. 51(1990), 69-91. MR.1025673(90k:47061)

[6] R. Curto and L. Fialkow, Recursively generated weighted shifts and the subnormal completion problem, Integral Equations Operator Theory, 17(1993), 202-246. MR.1233668 (94h:47050)

[7] - Recursively generated weighted shifts and the subnormal completion problem II, Integral Equations Operator Theory, 18(1994), 369-426. MR.1233668 (94h:47050)

[8] Solution of the truncated complex moment problems for flat data, Memoirs Amer. Math. Soc. 568(1996). MR1233668 (94h:47050)

[9] R. Curto, S. Lee and J. Yoon, k-Hyponormality of multivariable weighted shifts, J. Funct. Anal., 229(2005), 462-480. MR2183156

[10] R. Curto and W. Lee, Joint hyponormality of Toeplitz pairs, Memoirs of Amer. Math. Soc., Vol. 150, No. 712 (2001). MR1810770 (2002c:47042)

[11] M. Embry, A generalization of the Halmos-Bram condition for subnormality, Acta. Sci. Math.(Szeged) 35 (1973), 61-64. MR0328652 (48:6994)

[12] M. Embry and A. Lambert, Subnormality for the adjoint of a composition operator on $L^{2}$, J. Operator Theory, 25(1991), 309-318. MR.1203036 (94f:47028)

[13] G. Exner, On n-contractive and n-hypercontractive operators, Integral Equations Operator Theory, 56 (2006), 451-468.

[14] G. Exner, I. Jung, and S. Park, On n-hypercontractive operators, II, submitted.

[15] T. Furuta, Invitation to linear operators, Taylor \& Francis Inc., 2001. MR1978629 (2004b:47001) 
[16] M. Ito and T. Yamazaki, Relations between two inequalities $\left(B^{r / 2} A^{p} B^{r / 2}\right)^{r /(p+r)} \geq B^{r}$ and $A^{p} \geq\left(A^{p / 2} B^{r} A^{p / 2}\right)^{p /(p+r)}$ and their applications, Integral Equations Operator Theory, 44(2002), 442-450. MR.1942034 (2003h:47032)

[17] I. Jung, E. Ko, C. Li and S. Park, Embry truncated complex moment problem, Linear Algebra and Appl. 375(2003), 95-114. MR2013458(2004i:47030)

[18] I. Jung, C. Li and S. Park, Complex moment matrices via Halmos-Bram and Embry conditions, J. Korean Math. Soc., to appear.

[19] I. Jung and C. Li, A formula for k-hyponormality of backstep extensions of subnormal weighted shifts, Proc. Amer. Math. Soc. 129(2000), 2243-2351. MR1823917 (2002b:47061)

[20] A. Lambert, Hyponormal composition operators, Bull. London Math. Soc. 18(1986), 395-400. MR838810 (87h:47059)

[21] S. McCullough and V. I. Paulsen, A note on joint hyponormality, Proc. Amer. Math. Soc. 107(1989), 187-195. MR972236 (90a:47062)

[22] _ k-hyponormality of weighted shifts, Proc. Amer. Math. Soc. 116(1992), 165-169. MR:1102858 (93e:47029)

[23] M. Rao, Conditional measures and applications, Marcel Dekker, New York, 1993. MR 1234936 (95d:28001)

[24] J. Park and S. Park, On k-hyponormal weighted translation semigroups, Bull. Kor. Math. Soc. 39(2002), No. 4, 527-534. MR1938992(2003h:47042)

[25] J. Shohat and J. Tamarkin, The problem of moments, Math. Surveys I, Amer. Math. Soc., Providence, 1943. MR.0008438 (5:5c)

[26] R. Singh and J. Manhas, Composition operators on function spaces, North-Holland Math. Stud. No. 179, Amsterdam, 1993. MR.1246562 (95d:47036)

Department of Mathematics, College of Natural Sciences, Kyungpook National UniVERSiTy, DAEGU 702-702 Korea

E-mail address: ibjung@knu.ac.kr

Department of Mathematics, College of Natural Sciences, Kyungpook National UniVERSITY, DAEGU 702-702 KOREA

E-mail address: $1 \mathrm{mr} 67 @$ yumail.ac.kr

Institute of Mathematical Science, Ewha Womans University, Seoul, 120-750, Korea

E-mail address: pss4855@ewha.ac.kr 